\title{
Using acoustic reflex threshold, auditory brainstem response and loudness judgments to investigate changes in neural gain following acute unilateral deprivation in normal hearing adults
}

Hannah Brotherton

Manchester Centre for Audiology and Deafness, University of Manchester, Manchester Academic Health Science Centre, Manchester, M13 9PL, United Kingdom

hannah.brotherton@manchester.ac.uk

\section{Christopher J Plack}

Manchester Centre for Audiology and Deafness, University of Manchester, Manchester Academic Health Science Centre, Manchester, M13 9PL, United Kingdom

chris.plack@manchester.ac.uk

Department of Psychology, Lancaster University, Lancaster, LA1 4YF, United Kingdom

Roland Schaette

Ear Institute, University College London, London, WC1X 8EE, United Kingdom

r.schaette@ucl.ac.uk

Kevin J Munro

Manchester Centre for Audiology and Deafness, University of Manchester, Manchester Academic Health Science Centre, Manchester, M13 9PL, United Kingdom

Central Manchester University Hospitals NHS Foundation Trust, Manchester Academic Health Science Centre, Manchester, M13 9WK, United Kingdom

kevin.j.munro@manchester.ac.uk

Corresponding author: $\quad$ hannah.brotherton@ $\quad$ manchester.ac.uk ${ }^{1}$

\footnotetext{
${ }^{1}$ Present address: Department of Communication Sciences \& Disorders, University of South Florida, 4202 E. Fowler Avenue, PCD1017, Tampa, FL, United States of America, 33620-8200
} 


\section{ABSTRACT}

Unilateral auditory deprivation induces a reduction in the acoustic reflex threshold (ART) and an increase in loudness. These findings have been interpreted as a compensatory change in neural gain, governed by changes in excitatory and inhibitory neural inputs. There is also evidence to suggest that changes in neural gain can be measured using the auditory brainstem response (ABR). The present study extended Munro et al. (2014) [J. Acoust. Soc. Am. 135, 315-322] by investigating changes after 4 days of unilateral earplug use to: (i) ART, (ii) ABR and (iii) loudness. Because changes may occur during the post-deprivation test session (day 4), ART measurements were taken 1 hour and 2 hours post-earplug removal. There was a significant reduction in ART in the treatment ear immediately after the removal of the earplug, which is consistent with a compensatory increase in neural gain. A novel finding was the significant return of ARTs to baseline within 2 hours of earplug removal. A second novel finding was a significant decrease in the mean amplitude of ABR wave $V$ in the treatment ear, but a significant increase in the control ear, both after 4 days of deprivation. These changes in the ABR are in the opposite direction to those predicted. We were unable to replicate the change in loudness reported in previous deprivation studies; however, the short period of earplug use may have contributed to this null finding.

Key words: unilateral deprivation, neural gain, subcortical plasticity

Abbreviations: (ABR), Auditory brainstem response; (AN), auditory nerve; (ART), Acoustic reflex threshold; (BBN), Broadband noise; $(\mathrm{CN})$, Cochlear nucleus; (DCN), Dorsal cochlear nucleus; (ECV), Ear canal volume; (IC), inferior colliculus; (IHC), inner hair cells; (LL), lateral lemniscus; (NIHL), noise induced hearing loss; (SOC), superior olivary complex; (VCN), ventral cochlear nucleus. 


\section{INTRODUCTION}

Changes in the acoustic environment or hearing loss (real or simulated) can trigger a variety of adaptive mechanisms in the auditory brain. For example, a long-term reduction in auditory input can result in an increase in neural activity in response to a subsequent stimulus. This is achieved by scaling the strength of excitatory responses up and inhibitory responses down (Turrigiano, 1999). These changes have been investigated in humans using perceptual measurements such as loudness, and physiological measurements such as the acoustic reflex threshold (ART: the sound level required to elicit a contraction of the stapedius muscle in the middle ear) and the auditory brainstem response (ABR: an objective measure of neural activity in ascending auditory brainstem structures).

A change in loudness has been reported after using bilateral earplugs and noise generators (Formby et al., 2003; 2007). The change was observed in both ears and of a similar magnitude at 0.5 and $2 \mathrm{kHz}$, despite a difference in earplug attenuation at these frequencies. A similar pattern of change between the ears has also been reported after unilateral earplug use (Munro et al., 2014) and unilateral acoustic stimulation (Munro and Merrett, 2013). For example, using the categorical loudness test (Cox et al., 1997), Munro et al. (2014) reported a $5 \mathrm{~dB}$ decrease in sound level required to match pre-treatment loudness, at 0.5 and $2 \mathrm{kHz}$ in both ears of normal hearing listeners after 7 days of unilateral earplug use. These changes in loudness can be interpreted as a frequency-independent change in neural gain that occurs in both ears.

All studies that have investigated the ART after short-term unilateral deprivation have reported a different pattern of change in each ear. For example, Munro et al. (2014) reported a reduction in ART in the treatment ear after 7 days of unilateral earplug use in normal 
hearing listeners. In contrast, an increase in ART occurred in the control ear. This change was greatest at the high frequencies where the earplug provided greatest attenuation. A similar change was reported after increased unilateral acoustic stimulation (Munro and Merrett, 2013). The change in ART has been interpreted in terms of a compensatory change in neural gain operating at the lower level of the brainstem (Munro et al., 2014), which may attempt to counterbalance/reduce the asymmetry between the ears. ART measurements have not been made after bilateral changes in auditory input. Munro et al. (2014) also reported that most of the asymmetry between the two ears had disappeared within 24 hours of earplug removal.

The exact location of the physiological changes in the lower auditory brainstem remains unknown. Decker et al. (1981) were unable to demonstrate a significant change in the amplitude of the ABR following 10 to 30 hours of unilateral earplug use. However, as the deprivation period was relatively short, the ABR measurement may not have been sensitive enough to detect small changes in central neural gain. The authors did report a significant reduction in wave I latency. This is consistent with a decrease in neural conduction time at the level of the auditory nerve, which could be due to a change in the synchronous activity of neuronal populations (Skoe and Kraus, 2010), and is therefore not consistent with an increase in neural gain.

The present study extended that of Munro et al. (2014) by investigating ART, ABR and loudness after short-term unilateral deprivation. It was hypothesized that unilateral earplug use would induce a reduction in ART in the treatment ear and an increase in ART in the control ear. It was also hypothesized that there would be an increase in the amplitude of wave III and $\mathrm{V}$ of the $\mathrm{ABR}$ in the treatment ear and a decrease in the control ear. This would be consistent with a neural gain mechanism that operates at the level of the cochlear nucleus 
(CN), the main generator of wave III (Kaltenbach et al., 2000; Mulders and Robertson, 2009). The present study also measured loudness in order to investigate if changes could be observed over a shorter period of deprivation than that used in previous studies. It was hypothesized that loudness to a given test stimulus would increase after 4 days of unilateral earplug use, based on evidence of a change in loudness after 3 and 5 days by Munro and Merrett (2013). Finally, the ART was measured approximately 1 and 2 hours after earplug removal in order to characterise changes in neural gain within the post-deprivation test session.

\section{METHODS}

\subsection{Participants}

We calculated the sample size based on preliminary data from our laboratory. For the ART measurements, a power analysis revealed that 13 participants were required for a power of $80 \%$, assuming a within-subject difference of $4 \mathrm{~dB}($ s.d. \pm 6$)$ on a two-tailed paired samples $t$ test at 5\% significance level. For the ABR, we did not have a good estimate of effect size. Therefore, we based the sample size on previous ABR research by Schaette and McAlpine (2011) and $\mathrm{Gu}$ et al. (2012), which had sample sizes ranging from 15 to 21 . For the categorical loudness judgments, the analysis revealed that 28 participants were required for a power of $80 \%$, assuming a within-subject difference of $5 \mathrm{~dB}($ s.d. \pm 9$)$ on a two-tailed paired samples $t$-test at $5 \%$ significance level. Thirty-two participants initially took part in the study, which received ethics approval from The University of Manchester (ref: ethics/14261). However, four participants could not complete the study due to time constraints. As a result, 28 consenting volunteers (22 females and six males; median age, 22.5 years; range 19-50 years) participated in the study. Of those completing the study, 15 participants completed the ABR recording first and 13 completed loudness tests first. All participants were screened for 
normal hearing sensitivity [ $<20 \mathrm{~dB}$ hearing level (HL) from 0.25 to $8 \mathrm{kHz}$ and no asymmetry $>10 \mathrm{~dB}$ at any frequency] and normal middle-ear function on tympanometry (middle ear pressure +50 to $-50 \mathrm{daPa}$, middle ear compliance $0.3-1.5 \mathrm{~cm}^{3}$ ).

\subsection{Noise-attenuating earplugs}

The participants were fitted monaurally (14 left ear, 14 right ear) with a reusable Mack's silicone putty ear plug (McKeon Products, United States) and instructed to wear it continuously for 4 days, except for daily ablutions. The length of deprivation is based on the findings of Brotherton et al. (In press) where the change in ART in the treatment ear reached maximum 4 and 6 days after unilateral earplug use.

Sound attenuation of the earplug (i.e., the difference in ear-canal sound level with and without the earplug in situ) was measured using a clinical probe-tube microphone system. A calibrated probe tube microphone was inserted into the ear canal and the response to a $65 \mathrm{~dB}$ sound pressure level (SPL) pink noise signal was measured before and after inserting the earplug. The measures were made three times on each listener after the participant removed and refitted the earplug into each ear. The mean attenuation levels across the different fittings and the average attenuation level is shown in Fig. 1. The mean attenuation levels were 13-20 $\mathrm{dB}$ at $0.5-1 \mathrm{kHz}$ and $30 \mathrm{~dB}$ at $2-4 \mathrm{kHz}$. The attenuation values were similar to the levels reported by Munro et al. (2014). The mean difference between the attenuation values between each frequency were analyzed using paired $t$-tests. There were significant differences between 0.5 and $1 \mathrm{kHz}, 0.5$ and $2 \mathrm{kHz}$ and 0.5 and $4 \mathrm{kHz}(t(27.0)=-6.69, p<0.001 ; t(27)=-$ 18.02, $p<0.001 ; t(27)=-8.30, p<0.001$, respectively), and between 1 and $2 \mathrm{kHz}$, and 1 and 4 $\mathrm{kHz}(t(27.0)=-22.83, p<0.001 ; t(27.0)=-6.24, p<0.001)$, which survived after Bonferroni 
correction $(0.05 / 8)$ for multiple paired comparisons. There was no significant difference between 2 and $4 \mathrm{kHz}$ before and after a Bonferroni correction.

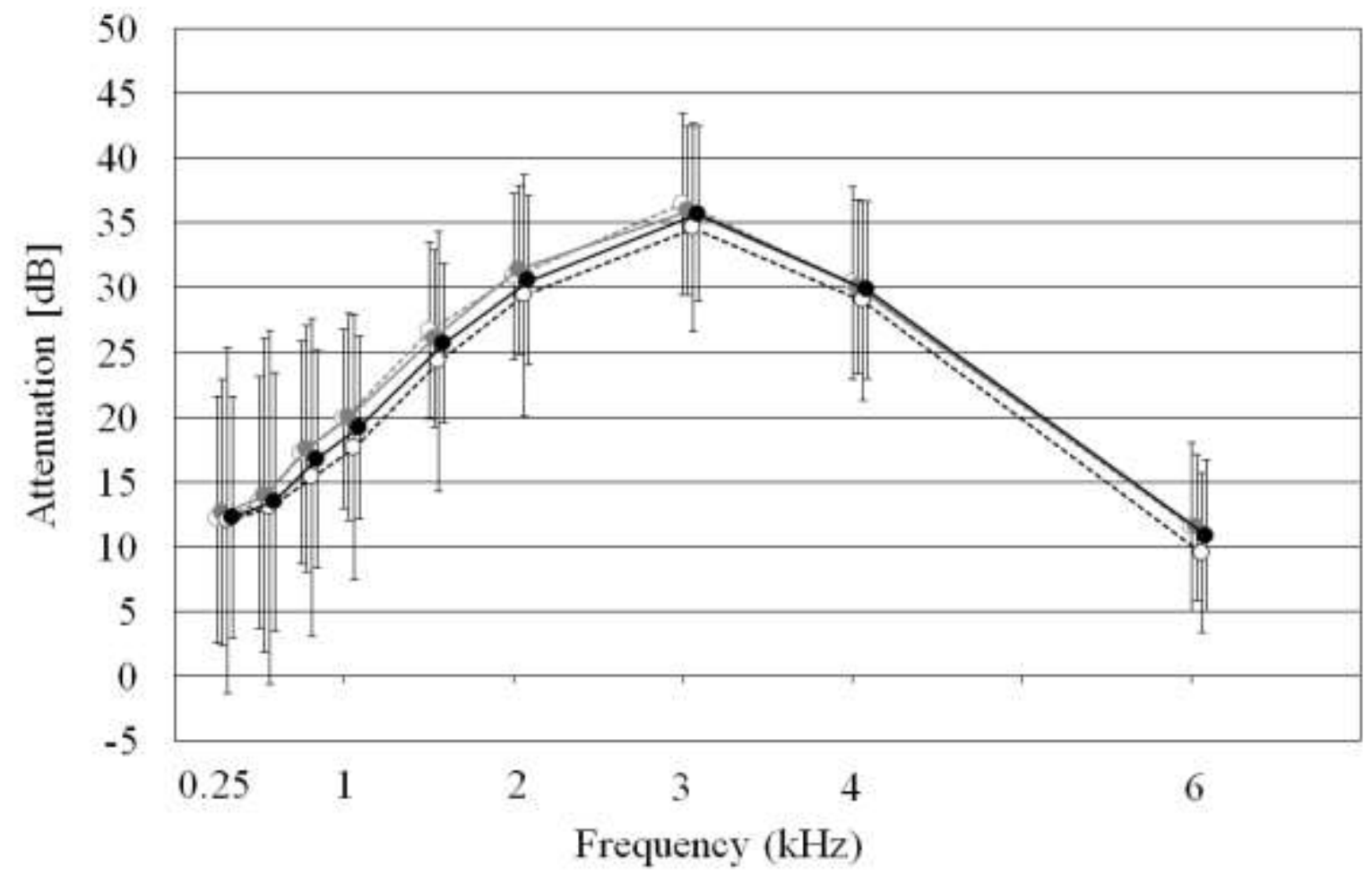

Fig. 1. Mean attenuation values taken on day 0 of earplug use for the first fitting (grey open circle with dotted line), second fitting (grey closed circle with solid line), third fitting (black open circle with dotted line) and the average attenuation values averaged across the three fittings (black closed circle with solid line). Errors bars show \pm 1 standard deviation $(n=28)$.

Although each participant was trained on how to insert the earplug into each ear, they were only fitted with a single earplug. The allocated ear was concealed from the researcher. This was achieved by asking each participant to choose a sealed envelope from a selection of envelopes, half of which contained instructions to wear the earplug in the left ear and the remaining half contained instructions to wear the earplug in the right ear. In addition, 
participants were asked to fit the earplug after leaving the test room on the first test session and to remove the earplug immediately before entering the test session room on the second test session.

\subsection{Equivalent ear-canal volume}

The equivalent ear canal volume (ECV), an estimate of the volume of air trapped between the probe tip and the tympanic membrane (Fowler and Shanks, 2002) was recorded. It is known that, for a given input, a smaller ECV would result in a higher sound level intensity, eliciting a reflex at an apparent lower level compared to a larger ECV. Because changes in ARTs could simply reflect a difference in ear canal insertion depth of the oto-admittance probe (i.e. a deep insertion depth after earplug use could result in a lower dial reading using the same sound level prior to earplug use), we recorded the equivalent ECV registered by the otoadmittance system. The mean ECV was $1.0 \mathrm{ml}( \pm 0.05)$ on both day 0 and 4 and in both the treatment and control ears.

\subsection{Physiological measures}

\subsubsection{Acoustic reflex thresholds}

Tympanometry was performed prior to measuring the ART immediately before and after 4 days of unilateral earplug use. ART measurements were always completed at the start of the test session. The ARTs were measured at these same times for the control ear. Preliminary data from our laboratory demonstrated a recovery of ARTs towards baseline levels 4 hours after the removal of the earplug. In the present study, the test sessions lasted around 2 hours, with the ABR and loudness measurements each taking around 1 hour to complete. Therefore, a change in neural gain may have occurred during the post-deprivation test session. As a 
result, ART measurements were made immediately after the completion of each test, i.e., after 1 and 2 hours, respectively.

Ipsilateral ARTs were measured using the GSI Tympstar middle ear analyser with a $226 \mathrm{~Hz}$ prove tone. Ipsilateral measurements involved presenting the eliciting stimulus and measuring the reflex in the same ear. The stimulus used to elicit a reflex was a broadband noise $(\mathrm{BBN})$. An investigation of treatment frequency specificity was not an aim of the present study. ARTs were included in the present study to confirm if any change in neural gain had occurred following 4 days of unilateral earplug use. BBN comprises the frequency range where the earplug had the maximum effect and has been shown to produce large, clear changes in ARTs following short term changes in auditory inputs (Brotherton et al., 2016). The stimulus was of $1 \mathrm{~s}$ fixed duration and presented at an initial level of $60 \mathrm{~dB}$ HL. The sound level was increased in $5 \mathrm{~dB}$ steps until the reflex was detected (reduction in compliance of $>0.02 \mathrm{~cm}^{3}$ ). Increasing the stimulus by a further $5 \mathrm{~dB}$ confirmed the reflex growth. The stimulus was decreased by $10 \mathrm{~dB}$ and increased in $2 \mathrm{~dB}$ steps to determine the ART. The stimulus was presented two additional times at the apparent ART to confirm repeatability and then increased by a further $2 \mathrm{~dB}$ to confirm reflex growth. If a change in compliance was not seen at the maximum stimulus eliciting level of $95 \mathrm{~dB}$ HL, $5 \mathrm{~dB}$ was added onto the maximum value as done in previous ART studies (Munro, Blount, 2009; Munro et al., 2014).

\subsubsection{Auditory brainstem response}

ABR measurements were recorded from all participants before (day 0) and after (day 4) earplug use using the NeuroScan System (STIM and SCAN). Disposable silver/silver chloride electrodes were placed in an array that consisted of a three-channel montage: vertex, 
ipsilateral and contralateral mastoids (positive), high forehead (ground) and the nape of the neck (negative). Electrode impedances were maintained at $<3 \mathrm{k} \Omega$. A $0.1-\mathrm{ms}$ alternating rectangular click was presented monaurally (in a balanced design) via ER-3A insert earphones at $80 \mathrm{~dB}$ normal $\mathrm{HL}$ (nHL; ca $110 \mathrm{~dB}$ peSPL) at a rate of 11.1 clicks/s. On-line analysis consisted of an artefact rejection ratio of $\pm 20 \mu \mathrm{V}$ and digital filtering from 30 to

$3000 \mathrm{~Hz}$. Off-line analysis was completed using Scan v4.5 (Neuroscan ${ }^{\mathrm{TM}}$ ) and consisted of referencing to the ipsilateral mastoid. The positive electrode remained as the vertex. An epoch window extending from $10 \mathrm{~ms}$ before and $15 \mathrm{~ms}$ after each click presentation was extracted. Artefact rejection was applied at $\pm 50 \mu \mathrm{V}$ and digital filtering from 150 to $1500 \mathrm{~Hz}$ (24 dB/Oct slope). Signals were averaged over 8000 sweeps and a linear detrend was applied to the data. Using Scan v4.5 (Neuroscan ${ }^{\mathrm{TM}}$ ), the peak-to-trough amplitude of waves I, III and $\mathrm{V}$ were identified using an automated detection algorithm for the maximum peak to the following minimum trough within a time window of 1-3, 3-5 and 5-8 ms, respectively. These values reflect a time window that has not been corrected for the time delay (around $0.8 \mathrm{~ms}$ ) introduced by the $256 \mathrm{~mm}$ tubing of the ER-3A earphones. The I:V amplitude ratio was also calculated. The peaks were checked visually to ensure that the peak-to-trough were clear within the time window. The peak data from five participants (randomly selected) were verified by a second investigator. We also took the opportunity to record the absolute latency values for wave I, III and V.

\subsection{Loudness measures}

The methodology for loudness measures was based on previous research (Munro and Merrett, 2013; Munro et al., 2014). Loudness judgments were obtained separately with $0.5-$ and $2-\mathrm{kHz}$ amplitude modulated pure tones using the Contour Test of Loudness Perception (Cox et al., 1997). The order of frequencies and ears was balanced between participants. Participants 
were instructed to assign one of seven loudness categories (very soft to uncomfortably loud) to a train of pulsed (1.5 s duration, inter-stimulus interval $2 \mathrm{~s}$ ) amplitude modulated tones (+5\% from the base frequency), which were presented monaurally via TDH-39 earphones with MX-41 cushion. The first train of pulsed tones was presented at $10 \mathrm{~dB}$ HL. After the participant allocated a loudness category to the stimulus, the sound level was increased by 5 $\mathrm{dB}$. The process was repeated until the participant allocated a sound level at the highest loudness category, i.e., uncomfortably loud. The process was carried out three times at each frequency in each ear. The median value of the sound levels for a given loudness response category was taken as the final value.

\section{STATISTICAL ANALYSIS}

The data were inspected before analysis to confirm that it was appropriate to use parametric statistics. Statistical analysis of the raw ART data was carried out using a two-factor (ear [2] $\mathrm{X}$ time [2]) repeated-measures analysis of variance (ANOVA). Statistical analysis of the ABR wave I, III and V amplitude data and the loudness data for the categorical loudness test were analyzed using a mixed ANOVA. Analysing each ABR wave separately simplified the interpretation of the results. For all analyses, the degrees of freedom were modified using the Greenhouse-Geisser correction when there was a statistically significant deviation from sphericity on Mauchly's test (Kinnea and Gray, 2009). All analyses were performed using SPSS version 22.

\section{RESULTS}

\subsection{Physiological measures}

\subsubsection{Acoustic reflex threshold}


The mean ARTs in the treatment and control ears before and after unilateral earplug use are shown in Figure 2. There was no difference between the two ears at baseline. After 4 days of unilateral earplug use, there was a reduction in the ART in the treatment ear and a small increase in the control ear. The difference between the ears after the earplug was removed was $7 \mathrm{~dB}$ but this had reduced to $5 \mathrm{~dB}$ at 1 hour post-earplug removal, and to $4 \mathrm{~dB}$ at 2 hours post-earplug removal.

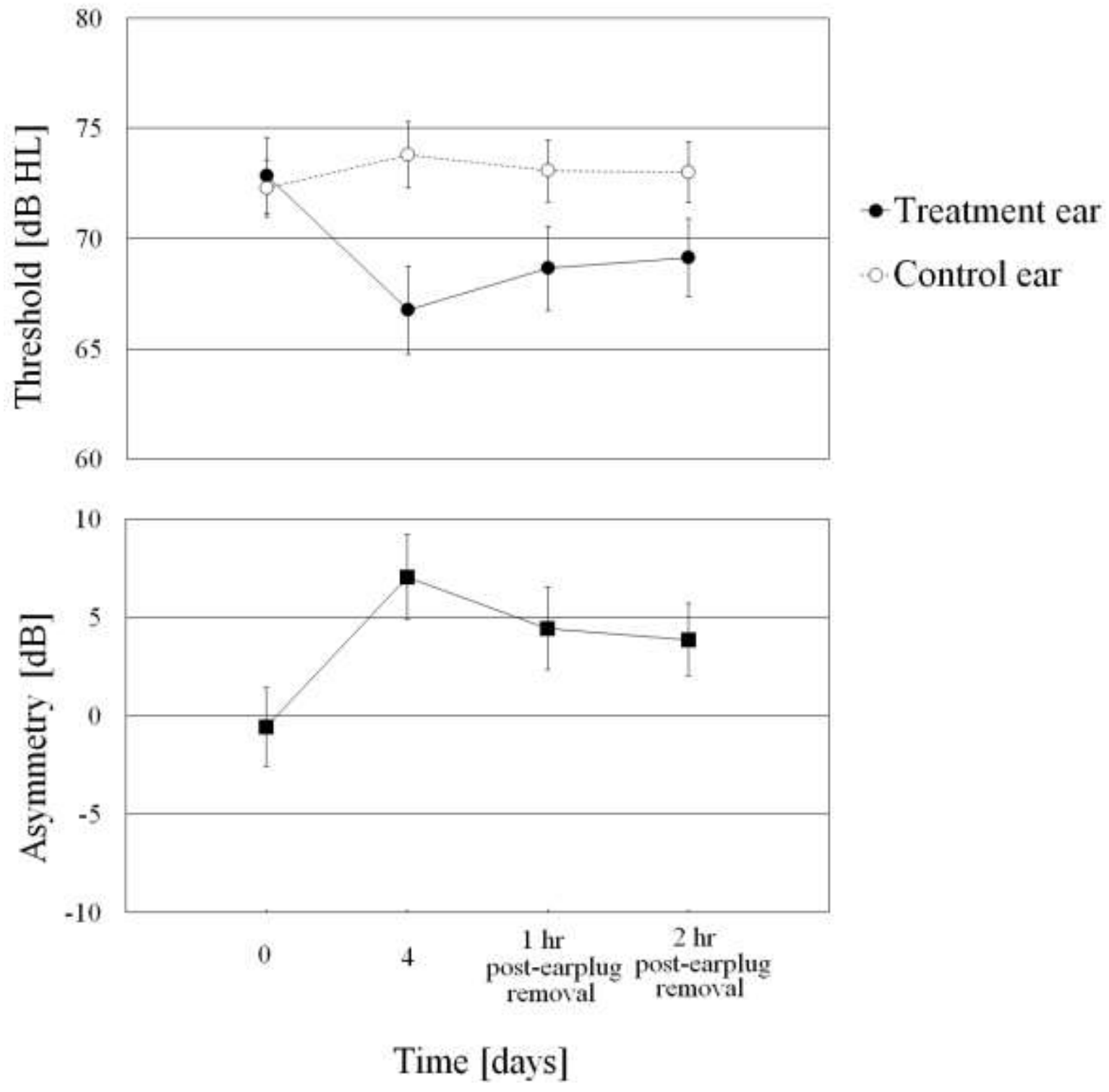


Fig. 2. Mean ART results using BBN stimuli. Top panel: Mean ART for treatment ear (filled circles) and control ear (open circles). Bottom panel: Difference between the control minus the treatment ear. Error bars show \pm standard error of the mean $(n=28)$.

In the two-way ANOVA, there was no effect of ear (treatment or control) on the ARTs, but there was a significant effect of time $[F(1,27)=7.675 ; p=0.010]$ and a significant interaction $[F(1,27)=20.866 ; p<0.001]$. Next, the data were analyzed for each ear separately using a one-factor (time [2]) repeated-measures ANOVA. There was no effect of time for the control ear, but there was an effect of time for the treatment ear $[F(1,27)=$ 27.095; $p<0.001$ ]. In summary, there was a significant $6 \mathrm{~dB}$ reduction in the treatment ear 4 days after unilateral earplug use.

Further analysis involved investigating the recovery of ART during the post-deprivation session, after the removal of the earplug on day 4. The mean difference between the ears at the three post-plug time points was analyzed using a one-way (time [3]) repeated-measures ANOVA. There was a significant effect of time $[F(2,54)=5.663 ; p=0.006]$. Paired sample $t$-tests were used to compare the mean difference between ears at each of the three post-plug time points. The difference between the ARTs at day 4 and 1 hour post-earplug removal was insignificant after Bonferroni correction $(0.05 / 3)[t(27)=2.464 ; p=0.020]$. There was a significant difference between the ARTs taken immediately after the removal of the earplug on day 4 , and 2 hours after earplug removal, after Bonferroni correction $[t(27)=3.174 ; p=$ 0.004]. There was no statistically significant difference between the ARTs taken 1 and 2 hours post-earplug removal, before or after Bonferroni correction. 
In summary, the mean ARTs for the treatment and control ears on day 0 and day 4 of earplug use were significantly different. After earplug removal on day 4, the mean ART showed a small recovery back towards pre-deprivation ART levels, which was significant 2 hours postearplug removal.

\subsubsection{Auditory brainstem response}

The mean ABR amplitude data are shown in Fig 3. There was no difference in the amplitude

of wave I, III, V and I-V amplitude ratio between the two ears at baseline. After 4 days of unilateral earplug use, there was a mean decrease in amplitude across waves in the treatment ear: wave I decreased by $21 \mathrm{nV}$, wave III decreased by $9 \mathrm{nV}$, wave $\mathrm{V}$ decreased by $35 \mathrm{nV}$ and the I-V amplitude ratio increased by $6 \mathrm{nV}$. The change in mean latency of wave I, III and V was negligible.

For the control ear, the changes in the mean peak-to-trough amplitude of wave I were variable: wave I decreased by $20 \mathrm{nV}$, wave III increased by $9 \mathrm{nV}$, wave V increased by $12 \mathrm{nV}$ and the I-V amplitude ratio increased by $11 \mathrm{nV}$, after 4 days of unilateral earplug use (see Fig. 3). The pattern of change for wave III follows the same direction as wave V: a decrease in the treatment ear and an increase in the control ear. The change in mean latency of wave I, III and V was negligible. 

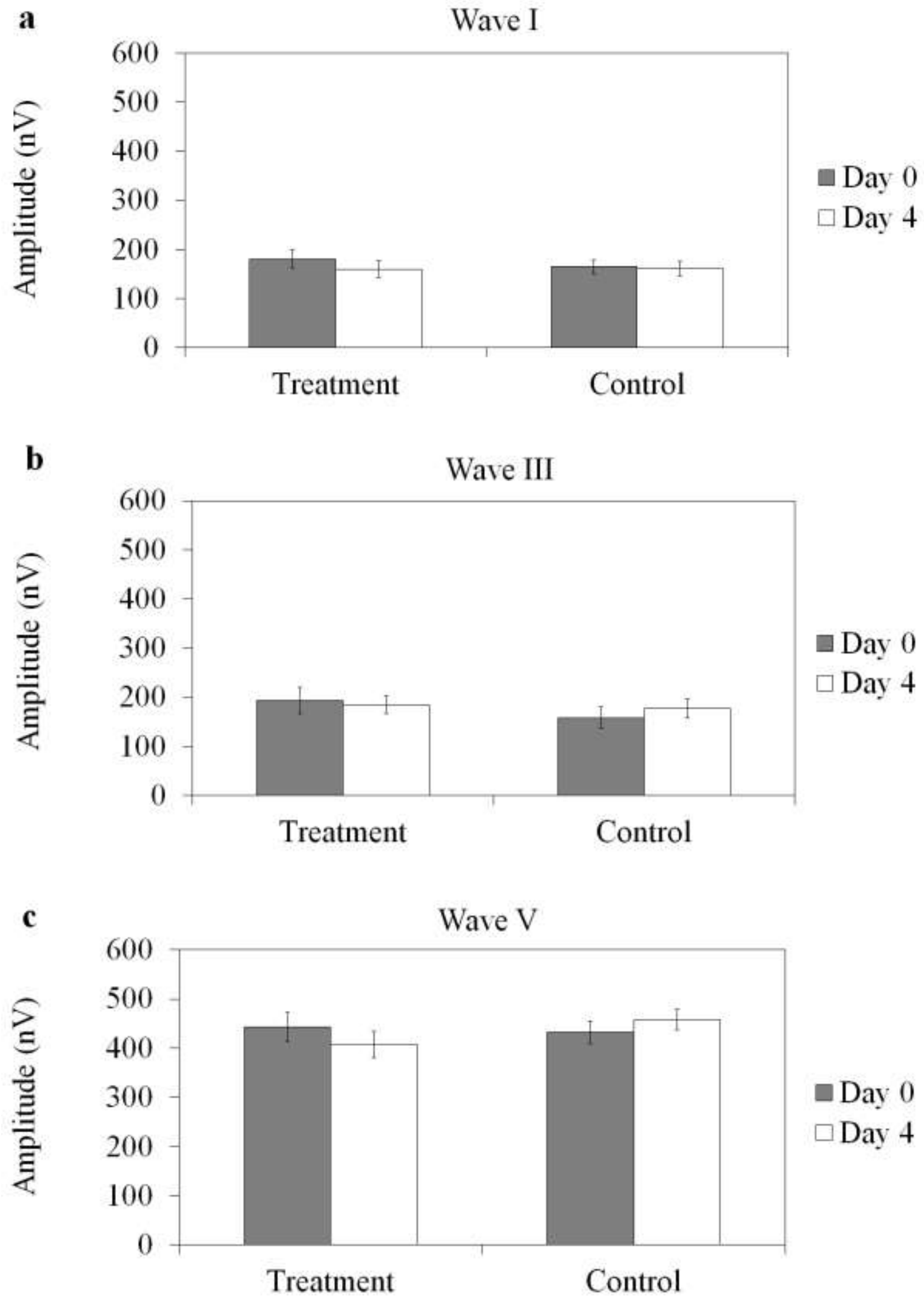
Fig. 3. Mean ABR data for the peak-to-trough amplitude of a) wave I, b) III and c) V for the treatment and control ears before (grey columns) and after (white columns) 4 days of unilateral earplug use. Error bars show \pm standard error of the mean $(n=28)$.

The peak-to-trough ABR amplitude data of waves I, III, V were analyzed separately using a three-way (time [2] X ear [2] X order [2]) ANOVA, with time (day 0 and 4) and ear (treatment and control ears) as within-subject factors and order (ABR/loudness first) as the between-subject factor. The ABR analyses were corrected for multiple comparisons $(0.05 / 3)$ using Bonferroni correction. There were no significant effect for wave I or I-V amplitude ratio. There were also no significant change in the mean latencies of wave I, III and V. For wave III, there was no effect of time or ear, and there was no interaction, but there was a significant effect of order $[F(1,26)=9.173 ; p=0.005]$ : The peak-to-trough amplitude of wave III was different if participants completed the ABR measurement first or second (see Fig. 4). The peak-to-trough amplitude of wave III (regardless of time and ear) was greater if this was measured after loudness testing. 

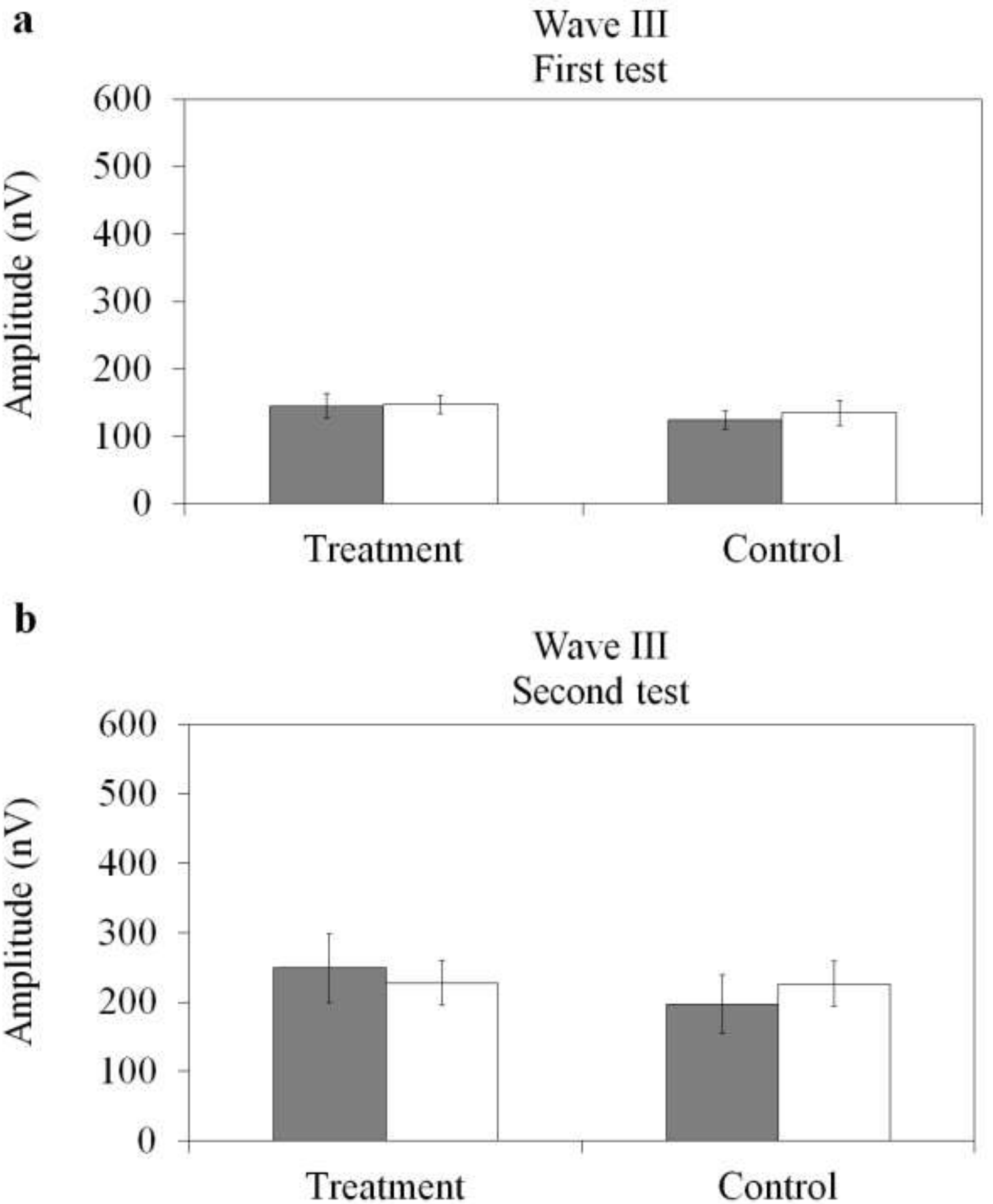

Fig. 4. The mean peak-to-trough wave III ABR data for participants that completed the ABR test first $(n=15)$ and second $(n=13)$. Error bars show \pm standard error of the mean. 
For wave $\mathrm{V}$, there was no effect of time, ear or order but there was a significant interaction between time and ear $[F(1,26)=7.697 ; p=0.010]$. We carried out a paired $t$-test on the difference in the peak-to-trough amplitude of wave $\mathrm{V}$ before and after earplug use for the treatment and control ear separately. There was no significant change in the wave $\mathrm{V}$ amplitude of the treatment ear. There was a borderline significant change in the wave $\mathrm{V}$ amplitude of the control ear $(t[27]=-2.076, \mathrm{p}=0.048)$, which did not survive after Bonferroni correction $(0.05 / 2)$ for multiple comparisons.

In summary, there was no significant change in the amplitude of wave I and I-V amplitude ratio over time. However, there was a significant increase in the amplitude of wave III but only when the ABR recording followed loudness measures. For wave V, the change in the treatment and control ear was significantly different after 4 days of unilateral earplug use: there was a reduction in the mean amplitude in the treatment ear and an increase in the control ear. However, the change over time for each ear was not significant.

\subsection{Loudness measures}


Treatment ear. $0.5 \mathrm{kHz}$

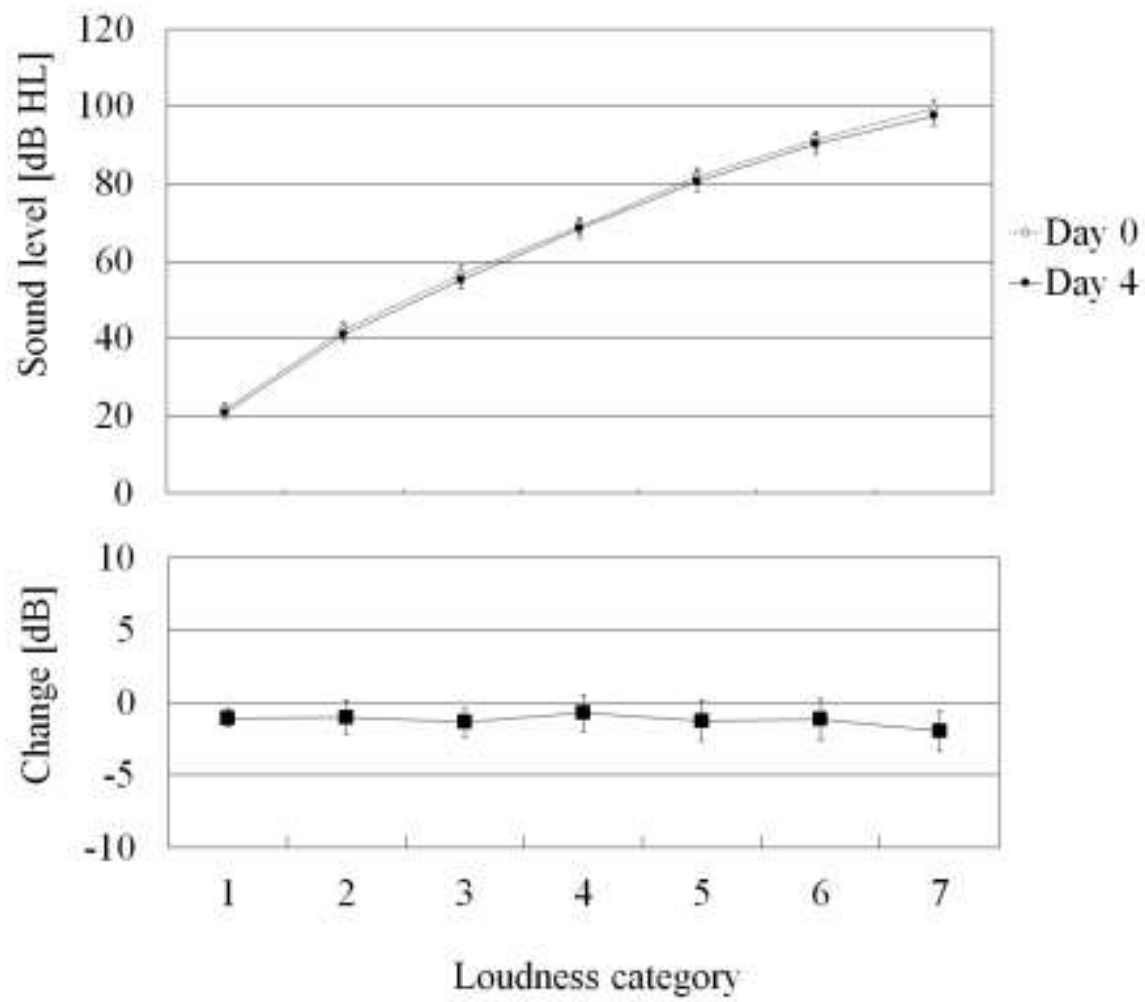

b Control ear, $0.5 \mathrm{kHz}$
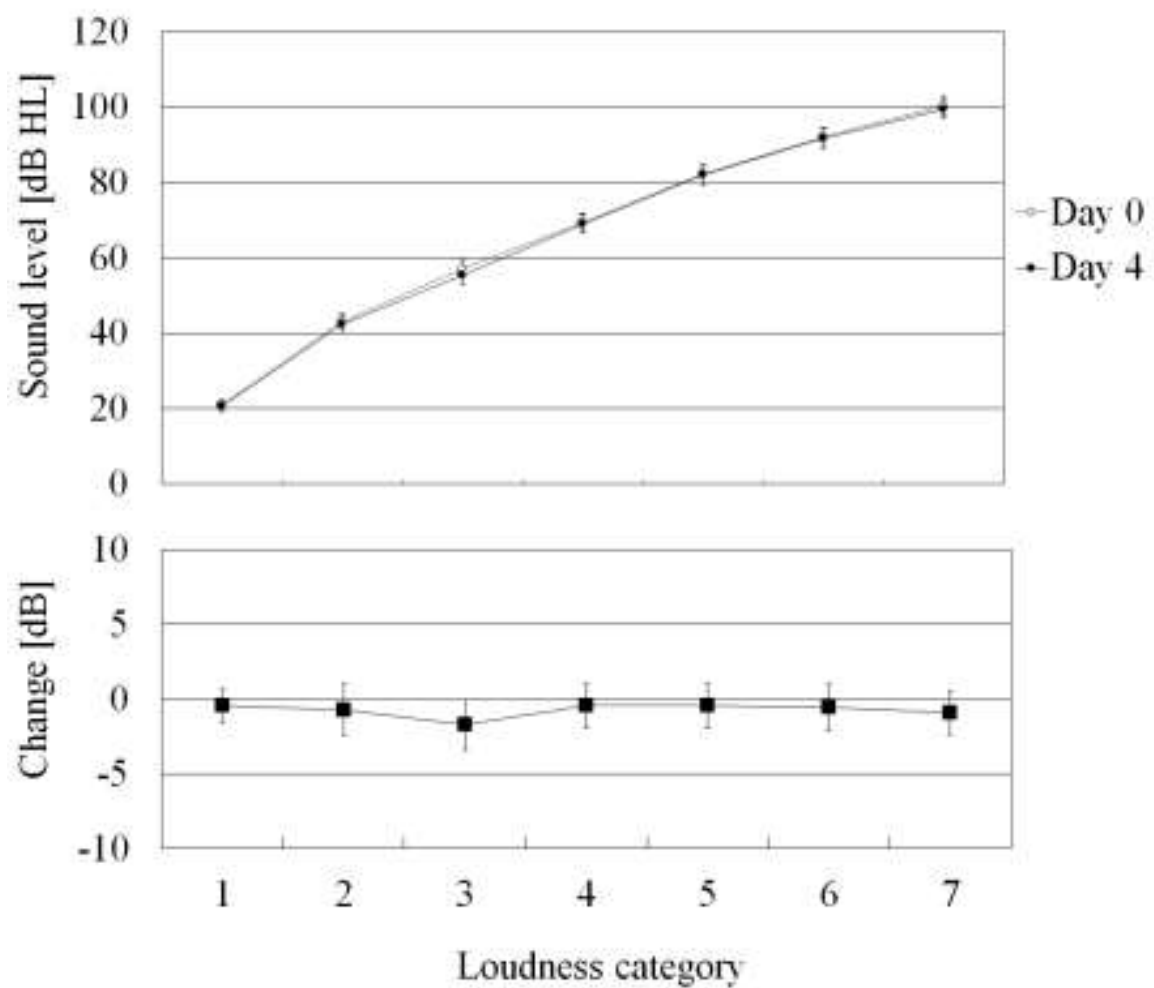
c Treatment ear, $2 \mathrm{kHz}$
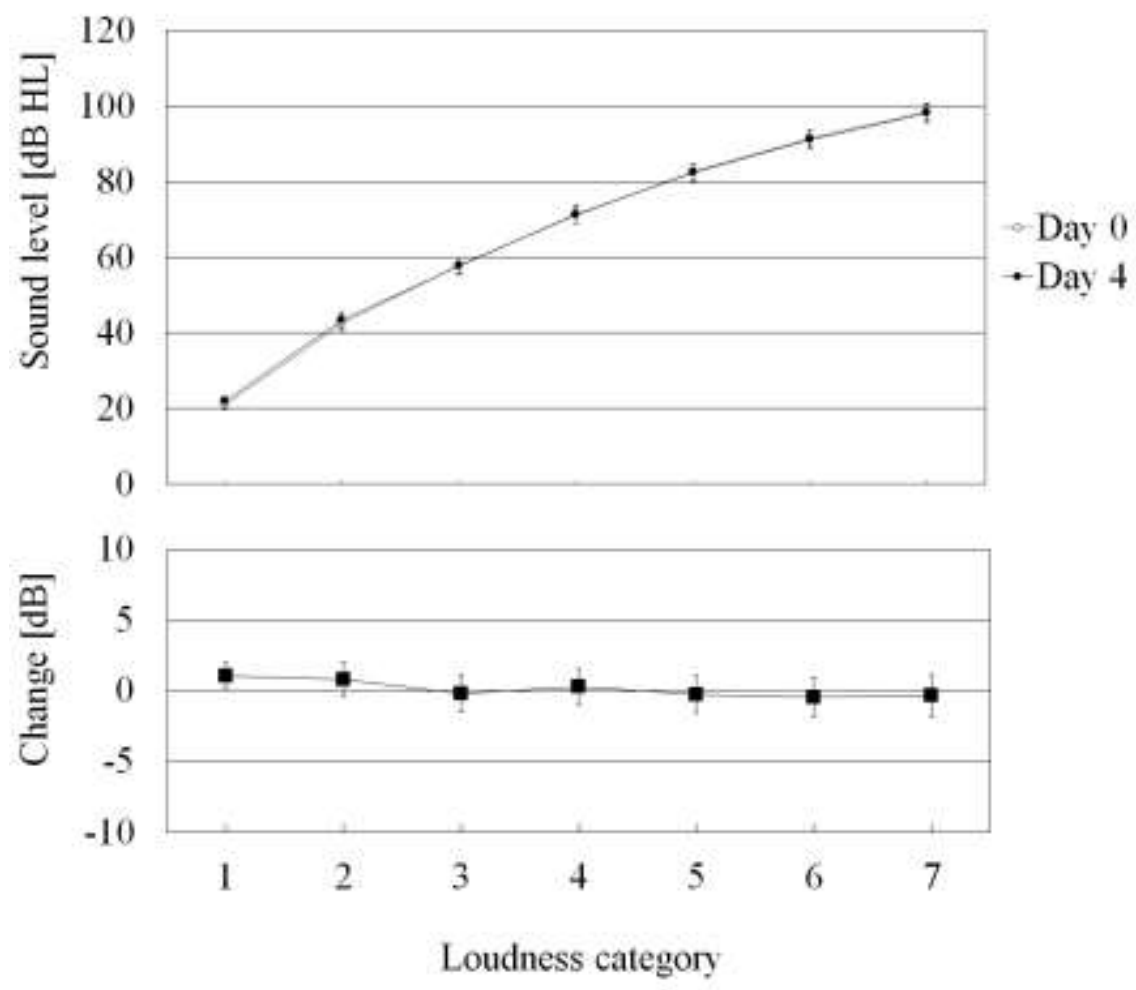

d $\quad$ Control ear, $2 \mathrm{kHz}$
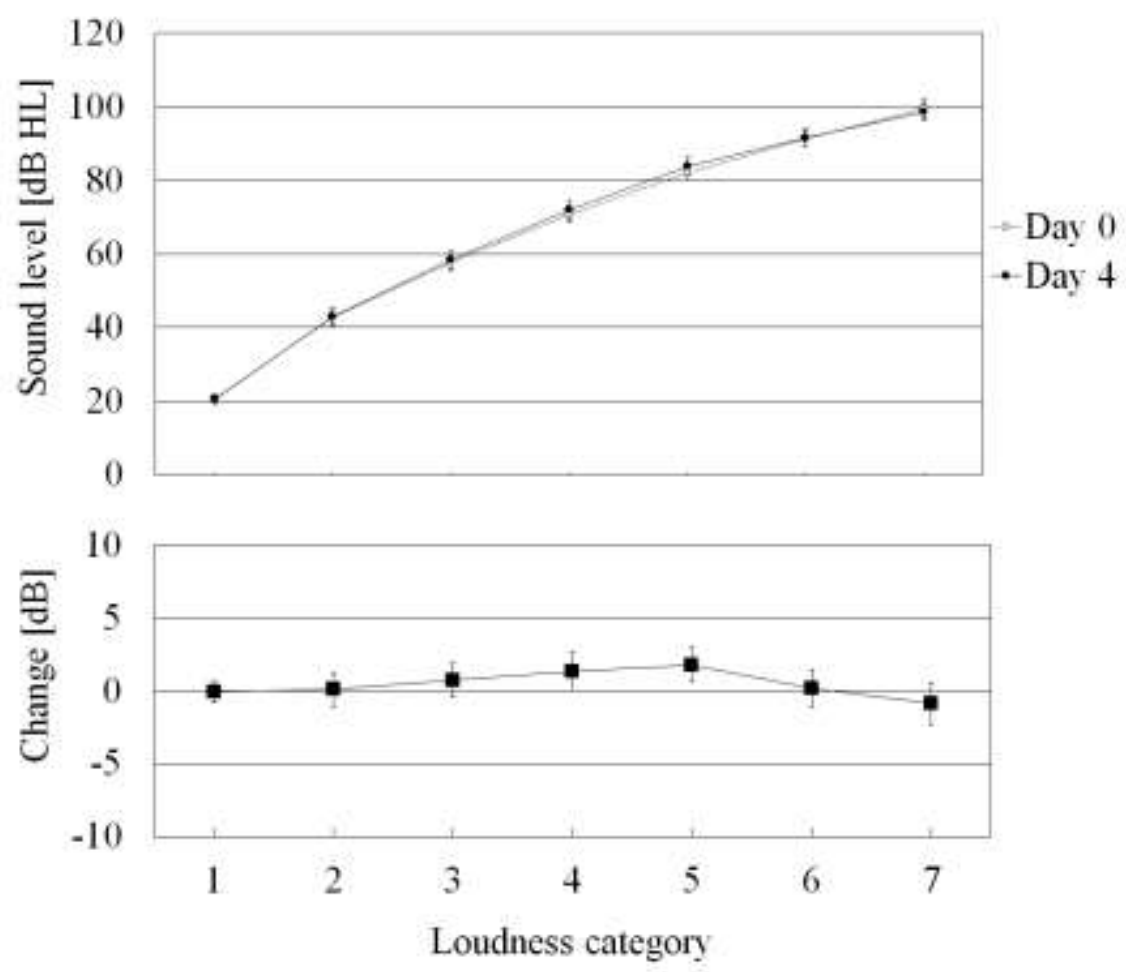
Fig. 5. Mean categorical loudness scaling results before (open circles) and after (filled circles) 4 days of unilateral earplug use. (a) Mean sound levels for the different loudness categories (top panel) and changes relative to baseline (bottom panel) for the treatment ear at $0.5 \mathrm{kHz}$. Dotted line with open circles, baseline; solid line with filled circles, day 4. All error bars show \pm standard error of the mean $(n=28)$. Loudness response categories: 1 , very soft; 2, soft; 3, soft/comfortable; 4, comfortable, 5, comfortable/loud; 6, loud; 7, uncomfortable. (b) Results for the control ear at $0.5 \mathrm{kHz}$, line styles and symbols as in (a). (c) Results for the treatment ear at $2 \mathrm{kHz}$, line styles and symbols as in (a). (d) Results for the control ear at 2 $\mathrm{kHz}$, line styles and symbols as in (a).

The mean sound levels at each loudness category are shown in Fig. 5. There was little change before and after treatment. For the categorical loudness test, the raw data were analyzed using a four-way (time [2] X ear [2] X loudness category [7] X order [2]) mixed ANOVA, with time (day 0 and 4), ear (treatment and control ears), loudness category (soft to uncomfortably loud) as within-subject factors, and order (ABR/loudness first) as the between-subject factor. The raw categorical loudness data were analyzed using a separate ANOVA for 0.5 and $2 \mathrm{kHz}$. The contour test analyses were corrected for multiple comparisons using Bonferroni correction (0.05/2). For $0.5 \mathrm{kHz}$, there was no effect of time, ear or order but there was a significant effect of loudness category $[F(1.547,40.220)=$ $710.952 ; p<0.001]$. For $2 \mathrm{kHz}$, there was a significant effect of loudness category $[F(1.560$, $40.567)=667.030 ; p<0.001]$. There was also an interaction of ear, time and loudness category $[F(3.476,90.376)=2.714 ; p<0.042]$, but this did not survive Bonferroni correction. In summary, the only significant finding for the $0.5 \mathrm{kHz}$ and $2 \mathrm{kHz}$ stimulus was the difference between each loudness category. 


\subsection{Correlation between variables}

We examined the correlation between the change in ART and the change in loudness in the treatment ear at 0.5 and $2 \mathrm{kHz}$ using the Pearson product moment correlation. We selected category 7 ("uncomfortably loud') for the loudness data as changes were expected to be greatest at high levels (Formby et al., 2003; 2007). There were no statistically significant correlations at 0.5 and $2 \mathrm{kHz}$.

\section{DISCUSSION}

\subsection{Physiological measures}

\subsubsection{Acoustic reflex threshold}

The present study revealed an asymmetry in ART of $7 \mathrm{~dB}$ between the two ears after 4 days of unilateral earplug use. This was primarily due to a reduction in ART in the treatment ear but there was also a small increase in the control ear. The magnitude and pattern of change in ART is similar to that reported by Munro and Blount (2009), Maslin et al. (2013b) and Munro et al. (2014). A slightly larger change in the control ear has been previously reported (Munro et al., 2014), which may be related to the longer period of deprivation.

The results are consistent with a compensatory change in neural gain after short-term monaural auditory deprivation: the earplug induced an increase in neural gain in the treatment ear and a decrease in neural gain in the control ear. Following noise induced hearing loss (NIHL) Cai et al. (2009) reported an increase in stimulus evoked activity in the ventral CN (VCN), which is part of the acoustic reflex arc. Therefore, it is likely that the neural gain underlying the changes in ART operates at the level of the VCN. This is supported by the 
results of Brotherton et al. (2016) who reported that the change in contralateral ARTs (stimulus in control ear) were not the same as the change in ipsilateral ARTs.

The opposite change between the ears could represent an attempt of the auditory system to maintain binaural asymmetry. A similar bilateral complementary change between the affected and unaffected ear has been demonstrated in the ABR following lesioning of the lateral olivary complex: a significantly higher wave I amplitude was observed ipsilateral to the lesion, compared to wave I contralateral to the lesion (Darrow et al., 2006).

The present study has demonstrated, for the first time, a significant level of recovery of ART within hours of removing the earplug. About $50 \%$ of the change in ART that occurred 4 days after earplug use had disappeared 2 hours post-earplug removal.

\subsubsection{Auditory brainstem response}

Since changes in ART suggest that the earplug has an effect on the lower brainstem, we hypothesized that these changes would also be seen as an increase in ABR waves III and V amplitude in the treatment ear, and a corresponding decrease in the control ear. In contrast, however, we observed a decrease in the amplitude of wave $\mathrm{V}$ in the treatment ear and an increase in amplitude in the control ear after a period of earplug use. This is consistent with a decrease in neural gain in the treatment and an increase in the control ear. This direction of change is not consistent with the pattern of findings reported in the majority of the animal studies. For example, spontaneous and stimulus-evoked activity has been shown to increase in the IC, when stimulating the ear that had been deprived of auditory input (Dong et al., 2010; Salvi et al., 1990). However, these data are single- or multi-unit recordings directly from the IC and may not be directly comparable to ABR measurements. In addition, these 
studies investigated changes in neural activity after noise trauma (Dong et al., 2010; Salvi et al., 1990). However, there are deprivation studies (involving transient conductive hearing loss) in animals that have shown effects that are consistent with the ABR findings in the present study (Hutson et al., 2008; 2009). For example, Hutson et al. (2009) reported a significant reduction in neural activity in adult gerbils (as measured by 2-deoxy-glucose uptake) 1 week after mild unilateral conductive hearing loss (removal of the malleus), in the afferent auditory pathway relative to the affected ear. Popescu and Polley (2010) also reported a reduction in the neural activity of the $\mathrm{CN}$, IC and $\mathrm{AC}$ following monaural unilateral ear canal ligation in adult rats. However, methodological differences such as the time of measurement (4 days versus 60 days post-treatment) prevents a direct comparison of the results from Popescu and Polley (2010) and the present study.

In humans, tinnitus has been associated with a reduced wave I amplitude but a normal (Schaette and McAlpine, 2011) or enhanced (Gu et al., 2012) wave V amplitude. These results are consistent with increased neural gain between the auditory nerve and central nuclei. However, these findings involve listeners with near-normal audiometric thresholds who may have 'hidden hearing loss', a selective loss of synapses with high-threshold auditory nerve fibers that is not detectable using pure tone audiometry (Plack et al., 2014). It is not clear that this is equivalent to the effects of earplug use.

We also observed an order effect on the amplitude of wave III in both the treatment and control ear. The mean amplitude was the same, relative to baseline, when participants completed the ABR before loudness measurements. In contrast, the peak-to-trough amplitude of wave III (regardless of time and ear) was greater if this was measured after loudness testing. The redistribution of ipsilateral and contralateral inputs in the treatment and control 
ear could also offer an explanation for the order effect of wave III amplitude. A recovery of ART occurred 1 hour after testing following earplug removal. Therefore, a reduction in neural gain during the first hour of testing, could present itself as an increase in the ABR amplitude when the measurement was conducted as the second test. Although wave III is generally discussed as representing the $\mathrm{CN}$ (Boston and Moller, 1985), it has also been suggested to represent contributions from the SOC (Chen and Chen, 1991).

\subsection{Loudness measures}

It was hypothesized that loudness would increase after 4 days of unilateral earplug use. However, there was a non-significant reduction of 1-2 $\mathrm{dB}$ in sound level required to match pre-treatment loudness judgments at $0.5 \mathrm{kHz}$ in both ears. The change at $2 \mathrm{kHz}$ was negligible. The current study was unable to replicate the significant reduction in loudness judgments at 0.5 and $2 \mathrm{kHz}$ reported by Munro et al. (2014) and Formby et al. (2003) after unilateral and bilateral earplug use, respectively. The present study used a shorter period of deprivation than these studies; therefore, the most likely explanation is that the length of deprivation was insufficient to induce a change. However, changes in processing when using binaural auditory tasks such as loudness lateralization (Florentine, 1976) or noise localization (Bauer et al., 1969) have been reported after a similar time scale of earplug use compared to the present study. The results are consistent with a change in loudness in the plugged ear. The sample sizes in the Florentine (1976) and Bauer et al. (1969) studies were very small, and McPartland et al. (1997) were not able to replicate Florentine (1976).

In the present study, a lack of change in loudness after 4 days of unilateral earplug use could suggest the existence of two distinct neural gain control mechanisms for loudness and ART. Previous studies have reported differences between the change in ART and loudness after 
unilateral change in auditory input: i) the change in loudness was observed in both ears, regardless of whether the treatment was restricted to one ear and, ii) the change in loudness was significant at lower frequencies despite the treatment providing little attenuation or increased stimulation at this frequency (Munro and Merrett, 2013; Munro et al., 2014). At present, there is no evidence to suggest a specific location in the auditory pathway at which there is a change in the neural gain control mechanism for loudness. However, if this mechanism is distinct from the acoustic reflex gain control mechanism, one can hypothesize that the neural gain control mechanism for loudness operates above the level of the acoustic reflex arc. This is consistent with reports that the temporal characteristics of neural gain can vary across different auditory structures (Syka et al., 1994), however this was reported between the IC and AC. It may be possible that a sufficient change in the neural gain control mechanism for loudness requires a longer period of deprivation compared to the change in in the neural gain mechanism underlying the changes in ART.

\section{CONCLUSIONS}

The ART findings from the present study are consistent with a compensatory neural gain control mechanism after 4 days of unilateral earplug use. The present study is the first to demonstrate a significant recovery of ART to baseline within 2 hours after earplug removal. This suggests that the decrease in neural gain is more rapid when input is restored, than is the increase in gain when input is deprived. In contrast, wave $V$ of the ABR showed a decrease in amplitude the deprived ear and an increase in the control ear. Finally, a lack of change in loudness after 4 days of unilateral earplug use could be due to a distinct neural gain mechanism separate to the mechanism underlying the change in ART. The loudness mechanism may require a longer time of deprivation for adaptive changes to occur. 


\section{DECLARATION OF CONFLICTING INTEREST}

The Authors declare that there is no conflict of interest 


\section{SUBMISSION DECLARATION}

All the authors have approved the final article 


\section{HUMAN RIGHTS}

Informed consent was obtained for experimentation with human subjects

The privacy rights of human subjects was always observed 


\section{FUNDING}

This work was supported by the University of Manchester 


\section{REFERENCES}

Bauer, R.W., Matuzsa, J.L., Blackmer, R.F. 1966. Noise Localization after Unilateral Attenuation. J. Acoust. Soc. Am. 40, 441-\&.

Boston, J.R., Moller, A.R. 1985. Brainstem auditory-evoked potentials. Crit. Rev. Biomed. Eng. 13, 97-123.

Brotherton, H., Plack, C.J., Schaette, R., Munro, K.J. 2016. Time course and frequency specificity of sub-cortical plasticity in adults following acute unilateral deprivation. Hear. Res. 341, 210-219.

Cai, S.Q., Ma, W.L.D., Young, E.D. 2009. Encoding Intensity in Ventral Cochlear Nucleus Following Acoustic Trauma: Implications for Loudness Recruitment. J Assoc Res Otolaryngol 10, 5-22.

Chen, T.J., Chen, S.S. 1991. Generator study of brainstem auditory evoked potentials by a radiofrequency lesion method in rats. Exp. Brain Res. 85, 537-42.

Cox, R.M., Alexander, G.C., Taylor, I.M., Gray, G.A. 1997. The contour test of loudness perception. Ear Hear. 18, 388-400.

Darrow, K.N., Maison, S.F., Liberman, M.C. 2006. Cochlear efferent feedback balances interaural sensitivity. Nat. Neurosci. 9, 1474-1476.

Decker, T.N., Howe, S.W. 1981. Short-Term Auditory Deprivation - Effect on Brain-Stem Electrical Response. Hear. Res. 4, 251-263.

Dong, S.Y., Mulders, W.H.A.M., Rodger, J., Woo, S., Robertson, D. 2010. Acoustic trauma evokes hyperactivity and changes in gene expression in guinea-pig auditory brainstem. Eur. J. Neurosci. 31, 1616-1628.

Florentine, M. 1976. Relation between lateralization and loudness in asymmetrical hearing losses. J. Am. Audiol. Soc. 1, 243-251.

Formby, C., Sherlock, L.P., Gold, S.L. 2003. Adaptive plasticity of loudness induced by chronic attenuation and enhancement of the acoustic background. J. Acoust. Soc. Am. $114,55-58$.

Formby, C., Sherlock, L.G.P., Gold, S.L., Hawley, M.L. 2007. Adaptive Recalibration of Chronic Auditory Gain. Semin Hear 28, 295-302.

Fowler, C., Shanks, J. 2002. In: Katz, J., (Ed.), Handbook of Clinical Audiology. Lippincott Williams \& Wilkins, Baltimore. pp. 175-204.

Gu, J.W., Herrmann, B.S., Levine, R.A., Melcher, J.R. 2012. Brainstem Auditory Evoked Potentials Suggest a Role for the Ventral Cochlear Nucleus in Tinnitus. J Assoc Res Otolaryngol 13, 819-833.

Hutson, K.A., Durham, D., Tucci, D.L. 2009. Reversible Conductive Hearing Loss: Restored Activity in the Central Auditory System. Audiol. Neurootol. 14, 69-77.

Hutson, K.A., Durham, D., Imig, T., Tucci, D.L. 2008. Consequences of unilateral hearing loss: Cortical adjustment to unilateral deprivation. Hear. Res. 237, 19-31.

Kaltenbach, J.A., Zhang, J.S., Afman, C.E. 2000. Plasticity of spontaneous neural activity in the dorsal cochlear nucleus after intense sound exposure. Hear. Res. 147, 282-292.

Kinnea, P.R., Gray, C.D. 2009. SPSS 16 made simple Psychology Press, Hove, UK.

Maslin, M.R.D., Munro, K.J., Lim, V.K., Purdy, S.C., Hall, D.A. 2013. Investigation of cortical and subcortical plasticity following short-term unilateral auditory deprivation in normal hearing adults. Neuroreport 24, 287-291.

McPartland, J.L., Culling, J.F., Moore, D.R. 1997. Changes in lateralization and loudness judgements during one week of unilateral ear plugging. Hear. Res. 113, 165-172.

Mulders, W.H.A.M., Robertson, D. 2009. Hyperactivity in the Auditory Midbrain after Acoustic Trauma: Dependence on Cochlear Activity. Neuroscience 164, 733-746. 
Munro, K.J., Blount, J. 2009. Adaptive plasticity in brainstem of adult listeners following earplug-induced deprivation. J. Acoust. Soc. Am. 126, 568-571.

Munro, K.J., Merrett, J.F. 2013. Brainstem plasticity and modified loudness following shortterm use of hearing aids. J. Acoust. Soc. Am. 133, 343-349.

Munro, K.J., Turtle, C., Schaette, R. 2014. Plasticity and modified loudness following shortterm unilateral deprivation: Evidence of multiple gain mechanisms within the auditory system. J. Acoust. Soc. Am. 135, 315-322.

Plack, C.J., Barker, D., Prendergast, G. 2014. Perceptual consequences of "hidden" hearing loss. Trends Hear 18.

Popescu, M.V., Polley, D.B. 2010. Monaural Deprivation Disrupts Development of Binaural Selectivity in Auditory Midbrain and Cortex. Neuron 65, 718-731.

Salvi, R.J., Saunders, S.S., Gratton, M.A., Arehole, S., Powers, N. 1990. Enhanced EvokedResponse Amplitudes in the Inferior Colliculus of the Chinchilla Following Acoustic Trauma. Hear. Res. 50, 245-258.

Schaette, R., McAlpine, D. 2011. Tinnitus with a Normal Audiogram: Physiological Evidence for Hidden Hearing Loss and Computational Model. J. Neurosci. 31, 1345213457.

Skoe, E., Kraus, N. 2010. Auditory Brain Stem Response to Complex Sounds: A Tutorial. Ear Hear. 31, 302-324.

Syka, J., Rybalko, N., Popelar, J. 1994. Enhancement of the Auditory-Cortex EvokedResponses Is Awake Guinea-Pigs after Noise Exposure. Hear. Res. 78, 158-168.

Turrigiano, G.G. 1999. Homeostatic plasticity in neuronal networks: the more things change, the more they stay the same (vol 21, pg 221, 1998) (vol 22, pg 280, 1999). Trends Neurosci. 22, 416-416. 